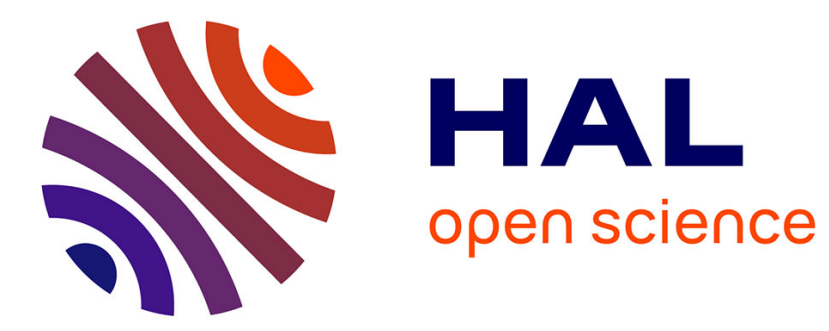

\title{
Quelques convergences remarquables entre l'Économie des Conventions et la Théorie de la Régulation
}

\author{
Franck Bessis
}

\section{To cite this version:}

Franck Bessis. Quelques convergences remarquables entre l'Économie des Conventions et la Théorie de la Régulation. Revue Française de Socio-Economie, 2008, 1, pp.9-25. 10.3917/rfse.001.0009 . halshs-00785370

\section{HAL Id: halshs-00785370 \\ https://shs.hal.science/halshs-00785370}

Submitted on 6 Feb 2013

HAL is a multi-disciplinary open access archive for the deposit and dissemination of scientific research documents, whether they are published or not. The documents may come from teaching and research institutions in France or abroad, or from public or private research centers.
L'archive ouverte pluridisciplinaire HAL, est destinée au dépôt et à la diffusion de documents scientifiques de niveau recherche, publiés ou non, émanant des établissements d'enseignement et de recherche français ou étrangers, des laboratoires publics ou privés. 


\title{
Quelques convergences remarquables entre l'Économie des Conventions et la Théorie de la Régulation
}

\author{
Franck Bessis $^{1}$
}

\section{Introduction}

La parution du recueil d'articles intitulé «La grande transformation de l'agriculture, Lectures conventionnalistes et régulationnistes », marque, en 1995, le point culminant des tentatives de mise en relation de deux des principales approches hétérodoxes françaises en cours de développement: l'Économie des Conventions (EC, par la suite) et la Théorie de la Régulation (TR, par la suite). Ces tentatives ont emprunté deux voies qui peuvent schématiquement être relues à partir des deux entrées de l'EC (Favreau, 1995) : i) pour l'entrée économique, la formalisation de la diffusion de nouvelles conventions ou de nouveaux compromis institutionnalisés à l'aide des outils de la théorie des jeux (Boyer et Orléan, 1994)², ii) pour l'entrée sociologique, l'investigation empirique des modalités d'émergence de compromis sur la qualification des biens et la prise en compte de la diversité des formes de coordination qui conduit à interroger, plutôt que de postuler a priori, l'existence d'une logique d'ensemble - tant du point de vue du mode de régulation, que de celui de chaque forme institutionnelle (Allaire et Boyer, 1995).

Dix ans plus tard, l'hypothèse d'un rapprochement souhaitable entre les deux approches est mise à mal par la «prise de conscience des différences entre les programmes de recherche régulationniste et conventionnaliste » (Boyer, 2004, p.111) argumentées par Amable et Palombarini (2005). Au cours de cette décennie, l'EC a marqué ses distances avec l'approche évolutionniste des conventions (Batifoulier, 2001) et, plus généralement, avec le cadre d'analyse de la théorie des jeux (Orléan, 2004) jugé inapte à rendre compte des dimensions interprétatives et politiques de l'action, en même temps que le programme s'est ouvert plus directement au fait institutionnel (Salais et alii, 1998), tout en maintenant l'attention portée aux jugements éthiques des agents (Bessy et Favereau, 2003), attention déjà présente au départ (Boltanski et Thévenot, 1991). De son côté, la TR a développé une analyse des représentations (Lordon, 1997) et du politique (Palombarini, 2001) mettant au premier plan les conflits d'intérêts et les rapports de domination en s'inspirant principalement de la théorie de l'action de Pierre Bourdieu (Boyer, 2003 ; Lordon, 2003 ; Reynaud, 2004).

\footnotetext{
${ }^{1}$ EconomiX, Université de Paris X - Nanterre, fr.bessis@gmail.com

${ }^{2}$ Bien que non revendiqué par ses auteurs comme une tentative de synthèse entre l'EC et la TR, ce travail a été discuté selon cette perspective par plusieurs commentateurs (voir notamment les réactions de Coriat (1994) et Lipietz (1995)). De ce fait, il a eu une influence non négligeable sur les débats entre les deux approches.
} 
De telles divergences renforcent le constat fréquent selon lequel les hétérodoxies partageraient bien peu de choses au-delà de leur opposition aux postulats néoclassiques. Contre ce constat, nous proposons de revenir dans cet article sur le sens originel du clivage entre orthodoxie et hétérodoxie tel que théorisé par Keynes. Ce sera l'objet de la première partie. La mise au premier plan de l'incertitude fournit une définition positive de l'hétérodoxie, promesse d'un socle commun. Celui-ci sera déployé dans les parties suivantes à partir d'une mise en parallèle des architectures théoriques de l'EC et de la TR. Cette présentation met en évidence, en amont de leurs divergences, les convergences essentielles entre les deux approches.

L'existence de divergences importantes à première vue, bien qu'elles ne soient pas traitées ici, nous invite à expliciter notre position. Nous visons un traitement symétrique des deux approches doublé d'un parti pris en faveur de leur unification. Cet article est la première étape d'un projet plus global visant à dessiner les linéaments d'un compromis acceptable par l'EC et la TR, parce que valant à la fois comme avancée pour chaque programme et extension de leur terrain d'entente. Pour ce projet, nous entendons moins départager ces deux courants que tirer avantage de leurs oppositions pour explorer leurs manques respectifs et proposer des amendements convergents qui soient, autant que possible, respectueux du niveau de cohérence des cadres initiaux et de leurs évolutions ultérieures ${ }^{3}$.

\section{La prise en compte de l'incertitude ou le versant positif de l'hétérodoxie}

Qu'entendons-nous précisément par hétérodoxie ? Pour préciser cette idée, nous pouvons nous appuyer sur sa figure à la fois la plus emblématique et la plus ambivalente : John Maynard Keynes. Olivier Favereau, qui s'est intéressé à son cheminement intellectuel, interprète les évolutions de la pensée et les ambiguïtés de l'œuvre de Keynes comme résultant d'une alternance entre deux projets, motivée par l'identification d'une économie « orthodoxe» durant la phase d'élaboration de la Théorie Générale. Sous l'influence de Wittgenstein ${ }^{4}$, il a découvert «dans la pratique de la théorie économique, non pas une investigation rationnelle de la vérité d'une réalité extérieure, mais un «jeu de langage », organisé autour de la production et de l'utilisation d'un langage formel (la «théorie économique ») avec ses règles et ses critères » (Favereau, 1988, p.201) et défini négativement par son incapacité à rendre compte du chômage involontaire tel qu'il fut expérimenté par les agents dans les années 1930. Ainsi les outils des «classiques» ne permettent pas de parler du chômage sans le dénaturer au regard du langage ordinaire et de l'idée que s'en font les agents à l'appui de leur

\footnotetext{
${ }^{3}$ Mentionner ces évolutions nous amène également à expliciter le niveau d'analyse retenu: il s'agit d'abord d'étudier les notions et orientations constitutives. A ce niveau, pour chaque approche, nous affirmons une forte cohérence entre leurs travaux les plus anciens et les plus récents. Ce parti pris est en accord avec les différentes synthèses de la TR fournies par Robert Boyer (notamment : 1986, 2004), qui reprend avec constance le même mode de présentation pour les notions de mode de régulation et de formes institutionnelles. Ce parti pris s'oppose du même coup à d'éventuelles lectures trop tranchées des distinctions du type «TR1/TR2 » et «EC1/EC2 » (Favereau, 1995, p.514).

${ }^{4}$ C'est l'hypothèse de Favereau (1985, 1988, 2001 et 2005).
} 
expérience vécue. La caractérisation négative de l'orthodoxie peut alors être généralisée par la formule suivante : « il y a un objet [le chômage] qui devrait être dedans - et qui est dehors » (Favereau, 2005, p.408). Partant de ce constat, la pensée de Keynes oscille entre deux projets. Le "projet pragmatique » (celui développé dans la Théorie Générale) vise l'amendement minimal de la théorie, en restant à l'intérieur du langage de l'orthodoxie, pour lui permettre d'intégrer le chômage involontaire. Le «projet radical» remet en cause le langage même de l'orthodoxie à partir de l'hypothèse de base fondamentalement responsable du problème, à savoir le rabattement de l'incertitude inhérente à la coordination sur du risque probabilisable.

L'explicitation de ce second projet keynésien est une caractérisation constructive de l'hétérodoxie : elle se définit par une prise en compte pleine et entière de l'incertitude (sous toutes ses formes) et de toutes ses conséquences. Au premier de rang de celles-ci figure la prise pour point de départ de l'analyse des défauts de coordination (ou encore des crises) plutôt que d'une logique de coordination parfaite. La non prise en compte des défauts de coordination, en réduisant l'organisation (institution ou entreprise) à une relation technique ou un ensemble de contrats et contraintes, empêche de parler de ce qui se passe à l'intérieur des organisations. Ce silence constitue une spécification possible de la nouvelle orthodoxie : il y a un objet, l'organisation, qui devrait être dedans et qui est dehors. Partir d'une coordination problématique en tenant compte de l'incertitude permet a contrario de parler, non seulement du chômage involontaire, mais aussi des entreprises et institutions comme dispositifs de coordination hors et entre marchés.

Les relectures de Keynes par Favereau et Orléan (1988) posent les jalons de la réactualisation de son projet radical et suggèrent la présentation suivante: le versant économique de l'EC est le développement du projet radical de Keynes. Développons ce constat. Les critiques adressées par l'EC à l'orthodoxie, à la différence de celles de Keynes, ne se concentrent plus principalement sur la manière dont celle-ci rend compte du chômage, mais portent, plus généralement, sur son traitement des défauts de coordination (Favereau, 2001). Les propositions constructives de l'EC généralisent, quant à elles, l'incertitude à l'ensemble des marchés. Plutôt que de limiter l'explication par les conventions de la détermination du taux d'intérêt sur le marché financier, le projet radical l'étend aux variables clefs des formes de "marché » plus éloignées du modèle walrassien idéal. L’hypothèse de rationalité limitée est le pendant microéconomique de cette généralisation de l'incertain. Elle permet, grâce à Simon, de donner un statut théorique à l'entreprise « en tant que forme d'adaptation collective aux limites cognitives individuelles » (Koumakhov, 2006, p.198).

La TR consiste, quant à elle, en une opérationnalisation, pour l'économie, de la lecture de Marx par Althusser (1965), et une mise en mouvement de ce structuralisme par une réintroduction d'une figure de l'agent permettant la restitution de la dimension créatrice des pratiques conflictuelles. Loin 
de présenter les rapports sociaux comme figés ${ }^{5}$, la TR insiste à l'inverse sur l'idée qu'ils contiennent une dynamique intrinsèque, en vertu de leur dimension contradictoire première par laquelle les individus sont à la fois unis et opposés dans leurs projets. Cette ambivalence est source d'une incertitude, directement intégrée au cadre marxiste revisité par la prise en compte du rapport marchand et l'institutionnalisation du sujet qui en résulte. La TR réintroduit ainsi également le message fondamental de Keynes, mais cette fois, au sein de l'orthodoxie structuraliste, tandis que l'EC part de l'orthodoxie «classique». Il en résulte l'idée d'une reproduction toujours problématique: une régulation. Dynamiser l'héritage d'Althusser en faisant de la régulation une reproduction non systématique, consiste ainsi, en partant du primat de la crise sur la reproduction, à fournir un traitement endogène des modalités de gestion, sans neutralisation complète, des contradictions contenues dans les rapports. Et la mise à jour du caractère problématique de la reproduction appelle ici encore le développement d'une théorie de l'action affranchie de l'idée de rationalité parfaite.

Une fois mise à jour la convergence autour du triptyque «incertitude radicale - défaut de coordination - rationalité limitée ${ }^{6} »$, nous pouvons rendre raison à d'autres similitudes importantes tant en amont qu'en aval de celui-ci.

En amont, la TR et l'EC sont d'abord guidées par une démarche réaliste. L'une des principales motivations originelles de la TR (de l'EC) est de construire une analyse des crises (des défauts de coordination) alternative à leur explication à partir de chocs exogènes ou d'écarts constatés entre le fonctionnement des économies réelles et le modèle de concurrence pure et parfaite. De cette perspective réaliste découle l'ouverture du cadre général à la nouveauté historique, soit à l'incertitude, à l'opposé d'un modèle dans lequel tous les états possibles du système sont connus par avance (Aglietta, 1976).

En aval, nous retrouvons les mêmes propositions alternatives à la théorie néoclassique. La critique $\mathrm{du}$ courant dominant apparaît alors comme seconde par rapport au projet constructif. Dans l'introduction collective au numéro spécial de la Revue Économique de mars 1989, les conventionnalistes présentent l'unité de leur démarche à partir du rejet de deux hypothèses de base du programme néoclassique.

Hypothèse 1: «les relations marchandes et les contrats d'échange entre les personnes les affranchissent de toute référence extérieure dépassant la rencontre de leurs volontés ».

Hypothèse 2: «le cadre néoclassique peut s'étendre continûment à l'analyse de relations non strictement marchandes ».

Ces deux hypothèses sont remplacées par les deux alternatives suivantes.

\footnotetext{
${ }^{5}$ La notion de rapport social, tout comme celle de convention, sera présentée au point 2.

${ }^{6}$ L'accord sur la reconnaissance des limites des capacités cognitives des agents (hypothèse de rationalité limitée comprise dans son sens le plus restrictif) n'empêche pas le désaccord sur la théorie de l'action à reconstruire à partir de ce postulat (voir notamment Servais (2000) et Postel et Sobel (2006)).
} 
Alternative 1: «l'accord entre des individus, même lorsqu'il se limite au contrat d'un échange marchand, n'est pas possible sans un cadre commun, sans une convention constitutive ».

Alternative 2: il existe «d'autres conventions constitutives, d'autres formes de coordinations étrangères au marché ».

Traduits dans le langage régulationniste, ces deux déplacements deviennent

Alternative 1': L'affirmation de l'existence d'institutions cachées nécessaires au fonctionnement d'une économie de marché (Boyer, 2004), au premier rang desquelles figure le système monétaire qui institue le sujet marchand (Aglietta et Orléan, 2002).

Alternative 2': En premier lieu, l'insistance sur une redéfinition de l'objet d'étude (dès les premiers travaux) - non pas l'économie marchande définie d'abord par des relations de concurrence, mais le capitalisme défini d'abord par des relations d'autorité -, en second lieu la relativisation du marché par l'affirmation d'au moins trois autres formes de coordination qui lui sont étrangères : l'Etat, l'organisation et le réseau (Boyer, 2003).

Le second déplacement a notamment pour application le passage d'une analyse en termes d'offre et demande de travail à une analyse en termes de règles de gestion de la main-d'œuvre (relation et rapport salarial pour la TR ou convention de qualité pour l'EC). Cette reprise de l'idée à la base de la notion de marché interne prend appui sur un message plus classique encore : le travail n'est pas une marchandise comme les autres. Pas plus que la mise au premier plan du rôle des variables institutionnelles, ce déplacement ne peut désormais être compté comme trait distinctif. Comme le souligne Favereau (1989) avec l'expression «théorie standard étendue », le cadre néoclassique s'est ouvert à l'analyse des formes de coordination alternatives au marché. Toutefois, même quand elle renonce à représenter l'entreprise comme un simple point de contact sans épaisseur entre des marchés, l'orthodoxie peine à restituer l'organisation comme entité collective et la réduit à un ensemble de contrats évalué de manière négative à l'aune des relations de concurrence parfaite au dénouement optimal.

Le premier déplacement continue, quant à lui, à servir de jalon au développement du projet radical. Il consiste d'abord en une remise au premier plan de la problématique classique de l'ordre social. Il comprend ensuite le rejet de l'hypothèse de nomenclature (Benetti et Cartelier, 1980) qui consiste à postuler l'existence d'une liste de biens définie a priori. En l'absence d'une telle hypothèse, la mécanique des arbitrages optimaux s'enraye faute de disposer d'un ensemble d'application complet et univoque. L'incertitude sur la qualité limite la rationalité au même titre que l'incertitude sur l'avenir et interroge les modalités de saisie des ressources contenues dans l'environnement. Tirer toutes les conséquences des limites des compétences cognitives des agents conduit également à rejeter l'hypothèse d'anticipations rationnelles pour s'intéresser à l'épaisseur du monde des représentations. La rationalité devient institutionnellement située dans un univers symbolique, au sein duquel le marché ne suffit plus à assurer de manière pacifique la compatibilité des plans individuels. Le conflit 
revient au premier plan et les deux courants sont ainsi amenés à souligner, selon des modalités variées, l'importance pour l'analyse économique de la dimension politique de l'action.

En résumé, ce que ces deux approches institutionnalistes ont de résolument hétérodoxe, est avant tout la mise au premier plan de cette incertitude radicale, qui les conduit à prendre pour point de départ de leur analyse les défauts de coordination (ou encore les crises) et une rationalité limitée. Dans la suite du texte, nous proposons de mettre en parallèle les architectures théoriques auxquels ce point de départ identique mène l'EC et la TR. Ces architectures concernent la manière dont chaque approche conçoit les régularités de comportements et d'attentes, conceptions dont découlent leurs notions constitutives (2.); les ancrages objectifs de ces régularités, dont découle leur conception des institutions (3.); et les cohérences entre ces règles et régularités, dont découle leur message sur la coordination (4.)

\section{Les régularités de pratiques et représentations comme régulation des} rapports sociaux et conventions

\subsection{Rapports sociaux}

Du côté de la TR, les régularités sont appréhendées à travers la notion constitutive de régulation, qui prend sens à la lumière des crises et de la reproduction des rapports sociaux contradictoires. Ces rapports sociaux sont des ensembles de comportements réguliers associés à la représentation de différents ensembles de places au sein d'un collectif. Cette définition rassemble deux manières de présenter la notion : i) comme régularité de comportement: "parler de rapports sociaux, c'est désigner la régularité de certaines pratiques sociales» (Lipietz, 1985, p.8); ii) comme classement: «Par rapport social on désigne tout ensemble de places en nombre défini ou indéfini (supérieur à deux), qui forment système en raison d'une codification établie à l'échelle de la société et dont certaines d'entre elles au moins sont similaires » (Billaudot, 1996, p.30) ${ }^{7}$. Expliciter le lien entre ces deux présentations permet de préciser la notion. Le rapport ne porte pas seulement sur les comportements - sur lesquels met l'accent la première présentation - mais bien également sur les représentations puisque « la reconnaissance sociale de la nature d'un rapport fait partie du rapport luimême » (Lipietz, 1985, p.9) - conformément à la seconde présentation.

Fait également partie du rapport la possibilité de sa reproduction, puisque celui-ci désigne des régularités. Il y a reproduction quand le résultat de la régularité ne remet pas en cause ses conditions d'apparition : dire qu'un rapport est «susceptible de se reproduire», c'est dire que «les pratiques qu'il combine socialement ont le double résultat de reproduire l'ensemble de leurs agents dans leur existence matérielle, et de les remettre en position d'accepter, ou d'imposer, de renouer les mêmes

\footnotetext{
${ }^{7}$ La codification permet une certaine stabilité des places ou encore une régularité de la représentation du (découpage du) collectif qui prévaut. C'est l'objet du point 3.
} 
rapports [...]. Les résultats des pratiques, dans le cadre d'un rapport, apparaissent identiques aux conditions de la mise en rapport » (Lipietz, 1985, p.10). Si ces conditions sont biens (re)produites par les pratiques, la logique de la reproduction n'en est pas moins dépendante des représentations ${ }^{8}$. Cette reproduction n'a cependant rien de systématique, car les rapports sociaux sont considérés dans leur contradiction, c'est-à-dire « comme unité (ou identité, etc.) et comme lutte (ou opposition, conflit...) de deux aspects (ou pôles, ou moments...) opposés» (Lipietz, 1979, p.27) selon une forme d'asymétrie (ou de domination) à spécifier. L'unité de la contradiction signifie que l'existence de chaque élément dépend de l'existence de l'autre - sans bourgeoisie pas de prolétariat et inversement. La dimension conflictuelle de la contradiction est considérée comme première ; ses aspects sont mis en rapport par la lutte. Cela apparaît clairement dans l'analyse marxiste, où le rapport bourgeoisie/prolétariat est avant tout présenté comme une lutte de classe entre exploiteurs et exploités. En conséquence, l'unité même du rapport repose sur la lutte, ce qui revient à affirmer le primat de la lutte sur l'unité. La reproduction n'est pas systématique, d'abord en raison de cette dimension conflictuelle toujours présente, qui contient un élément de dynamique interne au rapport, ensuite en raison des contradictions entre les rapports. Autrement dit, la régulation d'un rapport est sa reproduction toujours problématique du fait de son caractère contradictoire. Dans ces conditions, la crise «n'est que l'autre face de la régulation: l'une exprime, l'autre contient, la conflictualité originaire des rapports sociaux. » (Lipietz, 1985, p.14).

\subsection{Conventions}

Pour rendre compte des régularités, l'EC distingue quant à elle deux niveaux de conventions : convention $_{1}$ et convention $_{2}$. La convention $_{1}$ (ou modèle d'évaluation) est une représentation du collectif associée à une idée du fonctionnement correct de la relation que des individus forment entre eux. Elle se traduit ainsi simultanément par la construction d'un collectif (à l'état de représentation), l'affirmation d'une forme de coordination associée à une modalité d'évaluation prééminente en son sein, et la formation d'attentes sur les comportements respectifs de ses membres. Par les évaluations et attentes qu'elle soutient, elle contient une dimension normative mêlant à des degrés divers des considérations en terme d'efficacité et d'équité. La convention ${ }_{1}$ n'a pas le statut de méta-règle, mais celui d'hypothèse d'interprétation du collectif. Elle fournit une solution, sans régression à l'infini, au problème de l'incomplétude des règles, parmi lesquelles figurent les conventions $s_{2}$. Ces interprétations et attentes supposées partagées réduisent l'incertitude sans la neutraliser complètement; cette supposition sort renforcée de la réussite de la coordination sans jamais pour autant être garantie, d'autant que sa dimension normative ne l'exonère pas de conflits d'interprétations en vertu de la

\footnotetext{
${ }^{8}$ «Pour que les agents reproduisent des rapports, [...], [i]l faut d'abord qu'ils aient conscience que ce rapport peut exister (qu'il soit reconnu, même illusoirement, comme la " vente du travail »), et même qu'il apparaisse normal, naturel » (Lipietz, 1985, p.10).
} 
pluralité des principes d'actions et d'évaluations légitimes (les premiers n'étant pas réductibles au second) mobilisables dans toutes situations.

Les conventions $s_{2}$ (ou règles conventionnelles) correspondent initialement au sens courant du terme convention. Ce sont des règles implicites ou régularités observables. Elles partagent le plus souvent avec les conventions $s_{1}$ quatre caractéristiques : i) l'arbitraire ; ii) l'absence de sanction juridique ; iii) l'origine obscure; iv) l'absence de formulation précise ou officielle. Toutefois, seule la première

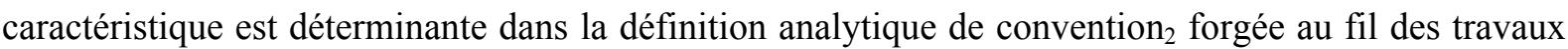
conventionnalistes (qui se démarque alors elle aussi, mais dans une moindre mesure, du sens courant). En tant que règles ou régularités, elles peuvent faire l'objet d'une formulation explicite, voire officielle, qui peut faciliter la reconnaissance de leur origine et même leur associer une sanction. Leur dimension conventionnelle ne repose crucialement que sur leur «logique du précédent, laquelle ne s'oppose pas tant à une logique du raisonnement (beaucoup de conventions sont raisonnables) qu'elle ne s'appuie sur une logique d'équilibres multiples » (Favereau, 2006).

\section{Les règles institutionnelles comme ancrages objectifs des régularités}

Explicitons à présent le passage de la dimension subjective des notions constitutives de conventions et de rapports sociaux à l'objectivité des règles et formes institutionnelles : les ancrages objectifs des régularités de comportements et d'attentes sont les supports matériels (dispositifs d'objets, règles écrites, lois, etc.) par lesquels les relations perçues subjectivement par les agents sont objectivées. Chaque règle institutionnelle est construite et interprétée au regard d'une convention ${ }_{1}$ ou d'un rapport social, mais elle a une réalité et des propriétés indépendantes de ses créateurs, susceptibles de faire l'objet d'une reconnaissance commune. C'est en ce sens que nous affirmons que les institutions fournissent un ancrage objectif aux conventions ${ }_{1}$ et aux rapports à partir desquels ils sont conçus, ce qui facilite leur partage.

\subsection{L'objectivation des rapports à travers leurs différents niveaux de codification}

Trois acceptions principales de la notion d'institution à l'œuvre dans la TR peuvent être retenues. Elles correspondent à trois niveaux de généralité des règles qui codifient les rapports : l'institution comme formes constitutives des rapports du mode de production; l'institution comme forme institutionnelle ; l'institution comme dispositif ou comme organisation qui correspond à l'acception commune du terme.

$\mathrm{Au}$ premier niveau, le mode de production désigne «toute forme spécifique des rapports de production et d'échanges, c'est-à-dire des relations sociales régissant la production et la reproduction des conditions matérielles requises pour la vie des hommes en société » (Boyer, 1986, p.43). Ces rapports, comme tout rapport social, contiennent une dimension symbolique (ou idéologique) et une dimension politique. Le mode de production combine ainsi symbolique, politique et économique. Le 
mode de production capitaliste spécifie la forme que prennent ces deux rapports sociaux fondamentaux dans une économie capitaliste : le rapport de production prend la forme salariale (on parle alors de rapport salarial), le rapport d'échange prend la forme marchande (rapport marchand). Pour une analyse circonscrite au capitalisme, ces deux formes, au niveau de généralité auquel elles sont spécifiée ici, ont le statut d'invariant structurel, c'est-à-dire de «relations sociales ayant atteint une maturité et pris des formes telles que les conflits dont elles sont le lieu et l'enjeu ne conduisent plus à leur remise en cause permanente mais à une lutte pour l'accès aux positions dominantes qu'elles déterminent » (Théret, 1992, p.58).

$\mathrm{Au}$ deuxième niveau, une forme institutionnelle se définit comme «toute codification d'un ou plusieurs rapports sociaux fondamentaux» (Boyer, 2004, p.39). L'État est à la fois une forme institutionnelle parmi les autres (qui détermine les dépenses et recettes publiques) et une forme présente à travers toutes les autres, en tant que principal vecteur de codification. Cette affirmation du rôle prépondérant du politique et de l'État par la médiation du droit a longtemps été sous-exploitée dans les travaux régulationnistes, d'abord soucieux de ne pas lui faire porter tout le poids de la réussite de la régulation. Celle-ci dépend autant des autres formes institutionnelles que des interventions de l'État. Toutefois, dans les changements de codification qui suivent les crises des formes institutionnelles et de la régulation, l'asymétrie entre l'État et les autres formes réapparaît: «les formes institutionnelles, qui ne sont autres que la codification des rapports sociaux fondamentaux, font intervenir, dans la quasi-totalité des cas, des décisions politiques relayées par le droit et la jurisprudence. L'État est donc l'un des vecteurs du changement institutionnel » (Boyer, 2004b, p.198).

La régulation caractérisant le moment de la cohérence, ou encore de l'absence de remise en question, les formes institutionnelles acquièrent, au sein d'un mode de régulation, le statut d'invariant structurel. Il est alors préférable, dans un souci de réalisme, d'expliciter un troisième niveau sur l'axe allant des notions les plus abstraites (le mode de production et ses rapports fondamentaux) aux règles concrètes. Autrement dit, les rapports sociaux fondamentaux du mode de production sont codifiés une première fois en rapport salarial et marchand, puis une deuxième fois en formes institutionnelles. Ce deuxième niveau de codification se précise encore dans des dispositifs susceptibles de varier à la marge sans que ces modifications ne se traduisent systématiquement par un changement des formes institutionnelles.

\subsection{L'objectivation des conventions à travers les opérations de mise en forme}

L'EC propose moins une hiérarchie des codifications qu'une hiérarchie des conventions selon leur plus ou moins grande capacité d'extension en lien avec l'architecture des régimes d'engagement développée par Thévenot (2006). Parce qu'ils ne comportent pas de hiérarchie alternative, mais affinent l'idée de codification à partir de celle de mise en forme, précisant ainsi les propriétés cognitives de l'objectivation des conventions ou rapports dans des règles, les développements de l'EC 
peuvent ici compléter ceux de la TR. L'opération de mise en forme consiste à stabiliser une coordination locale et ponctuelle en la faisant passer d'une forme singulière à une forme, au moins transposable à d'autres situations, voir généralisable à toutes les situations. La mise en forme d'une coordination suppose la mise en forme ou qualification d'objets (ou de personnes), qui consiste à établir l'accord sur la qualité d'une chose (ou d'une personne) à partir de sa mise en équivalence avec d'autres choses ou personnes, selon le principe d'évaluation d'une convention ${ }_{1}$. La présence d'objets qualifiés dans une situation rend plus évident le recours à la forme de coordination qui leur est associée

La notion d'investissement de forme (Thévenot, 1985 et 1986 ; Eymard-Duvernay, 1986) rend compte du coût que suppose cette opération de passage d'une chose indéterminée ou régularité observable à un objet (une qualité) qualifié(e) ou une règle explicite ${ }^{9}$. L'élaboration d'un label de qualité est un exemple d'investissement de forme. Il permet de créer une équivalence entre des produits différents en privilégiant le respect de certains critères de fabrication. Il s'oppose aux critères d'évaluation de la personne qui privilégie les conseils de son entourage, ou la fidélité à une marque. Ces autres formes d'équivalence mettent en jeu des investissements d'une autre nature basés sur l'établissement d'une relation de confiance ou des efforts de publicité.

Les dispositifs sont le produit direct d'investissements de forme et produisent à leur tour la généralité d'une forme. Par exemple, une règle prend sens à la lumière du système métrique et permet de produire des qualifications d'objets, identifiables par tous, selon leur taille. Ils permettent d'alléger la charge cognitive requise des personnes pour la coordination en leur servant d'équipements extérieurs mobilisables dans leurs opérations de jugement. Ainsi, « les compétences d'association sont ancrées sur des dispositifs » (Eymard-Duvernay, 1999, p.165). Il existe des dispositifs de toute taille les conventionnalistes parlent de «microdispositifs» ou de « macrodispositifs » (Eymard-Duvernay, 2006, p.13) - par lesquels varie le niveau de la coordination et le degré produit de généralité de la forme.

\section{Cohérences}

Comment les deux approches conçoivent-elles désormais la cohérence entre la pluralité des régularités de comportements et d'attentes et leurs ancrages objectifs? Nous tâchons maintenant de poursuivre leur mise en parallèle en apportant des éléments de réponse à cette question à la fois au niveau macro et microéconomiques.

\footnotetext{
${ }^{9}$ «Investir, c'est établir, par l'effet d'une dépense à un instant donné, ou par l'effet cumulé dans le temps d'une habitude, la validité d'une forme sur une durée et un domaine de validité déterminés » (Eymard-Duvernay, 1986, p. i).
} 


\subsection{Cohérence globale : mode de régulation et équilibre de règles}

Le mode de régulation consiste dans «tout ensemble de procédures et de comportements, individuels et collectifs, qui ont la triple propriété : de reproduire les rapports sociaux fondamentaux du mode de production à travers la conjonction de formes institutionnelles historiquement déterminées; de soutenir et «piloter» le régime d'accumulation en vigueur; d'assurer la compatibilité dynamique d'un ensemble de décisions décentralisées, sans que soit nécessaire l'intériorisation par les acteurs économiques des principes de l'ajustement de l'ensemble du système » (Boyer, 2004, p.54). Cette définition canonique distingue bien les règles codifiées des procédures et comportements. En tant que codifications de rapports sociaux, les formes institutionnelles répondent à la définition générale des institutions comme système de règles. Les procédures et comportements opèrent sur les rapports sociaux et comptables par la conjonction de règles, dont elles se distinguent donc, sinon systématiquement en pratique, du moins d'un point de vue analytique. Ainsi, la notion de mode de régulation témoigne de la compatibilité d'un ensemble de règles ou de formes institutionnelles, mais se compose de l'ensemble de procédures et de comportements situés dans (et en cela cognitivement orientés par) ces règles, tout en en étant distincts.

L'équivalent de cette notion dans le langage conventionnaliste est celle d'équilibre de règles. Il s'agit d'un "point fixe sur les schémas de révision de transactions »: «les règles d'adaptation et d'ajustement des actions peuvent entraîner des rectifications dans les propositions de transactions, mais ces modifications s'effectuent selon des schémas qui ne sont pas remis en cause par l'observation des nouvelles transactions réalisées » (Favereau et Thévenot, 1996, p.308). Cette notion est mobilisable à différents niveaux: organisation, branche, marché, société ou macroéconomie. L'équilibre, qui porte sur des règles ou conventions, ${ }_{2}$, est dynamique. Il admet, à tout moment, des désajustements - ratés dans le processus de production (retard de commande, pièces défectueuses, instructions contradictoires, etc.) déséquilibres de transactions (chômage, stock, pénurie, etc.) - que ces règles visent précisément à gérer, conformément à une représentation, dont ces désajustements et réactions ne remettent pas en cause le caractère partagé posé à titre d'hypothèse (ce qui en fait une convention $_{1}$ ). Il formalise ainsi, par un ensemble de conventions ${ }_{2}$, l'hypothèse corroborée d'un accord sur le fonctionnement d'un collectif.

Mettant en parallèle ces deux notions, nous confortons une hypothèse d'articulation entre les deux approches émise par Robert Boyer : « les idées forces des conventionnalistes pourraient s'appliquer à la macroéconomie conçue comme un équilibre de règles et de formes d'organisation [...]. $\mathrm{Au}$ demeurant, c'est retrouver l'inspiration initiale des modèles macroéconomiques régulationnistes qui examinent [...] la compatibilité [...] de compromis institutionnalisés qui ne font que codifier des principes partiels et qui doivent faire la preuve de leur acceptabilité grâce à la viabilité du mode de régulation qu'ils sous-tendent » (Boyer, 1995). 
Parvenu à cette remarquable convergence, il est temps de rappeler l'opposition principale entre les deux approches qui ne sera pas interrogée ici - d'abord parce qu'elle déborde l'objet de cet article (dont l'objectif est, rappelons-le, de fournir une présentation positive du socle commun à l'EC et la TR), ensuite faute de place. Tandis que l'EC, par l'intermédiaire des conventions, met en avant la saisie normative des institutions par les agents, les régulationnistes conçoivent les formes institutionnelles comme résultant de compromis institutionnalisés (Delorme et André, 1983) établis sur fond de rapports de force, d'abord indépendamment de toutes considérations en termes de légitimité. Les deux approches font ainsi reposer la dynamique des institutions de manière cruciale sur des processus d'ordre politique, mais elles semblent concevoir chacune ce moment politique de manière contrastée : les conventionnalistes le présentent avant tout comme le moment d'un arbitrage délibéré dans la pluralité des formes de coordination alors que les régulationnistes le conçoivent d'abord comme celui d'un arbitrage imposé entre des intérêts divergents. Il manque donc ici l'articulation des registres cognitifs (développés en 3.) et politiques entendu dans les deux sens du terme (imposition et co-élaboration sous contrainte de légitimité des règles).

Malgré cette limite, il est possible de réaliser une étape supplémentaire dans la mise à jour des points d'accord entre l'EC et la TR. Celle-ci porte sur l'entreprise. Loin de relever d'une stratégie de repli face aux difficultés précédentes, ce déplacement de l'analyse vers un espace de coordination plus restreint révèle une dernière convergence tout à fait significative. Cette dernière pourrait en effet servir de point de départ au traitement de l'opposition mentionnée, dans la mesure où le projet radical de Keynes, à la lumière duquel cette institutionnalisme prend sens, peut s'entendre comme « une pensée de l'économie à partir de l'entreprise » (Favereau, 1988, p.214).

\subsection{Cohérence locale : modèles productifs et modèles d'entreprise}

Au niveau de l'entreprise, l'accumulation repose pour la TR sur l'adaptation à un mode de croissance d'une stratégie de profit mise en œuvre par un modèle productif défini comme un compromis de gouvernement d'entreprise ${ }^{10}$. Le mode de croissance est caractérisé par une source principale du revenu national (investissement, consommation intérieure ou exportations) et une forme de distribution de ce revenu. De manière plus large, cette notion de mode de croissance nous semble devoir être comprise comme la perception par les agents de la conjonction d'un régime d'accumulation (qui est une construction abstraite du théoricien) et d'un mode de régulation (les régulationnistes parlent encore de mode de développement). Perception sans laquelle les agents ne pourraient pas adapter leurs stratégies de profits. Ces stratégies de profit sont des «combinaisons

\footnotetext{
${ }^{10}$ «Les modèles productifs peuvent être définis comme des « compromis de gouvernement d'entreprise », qui permettent de mettre en œuvre durablement et avec profit une des stratégies de profit viables dans le cadre des modes de croissance des pays où les firmes organisent leurs activités, grâce à des moyens (politique-produit, organisation productive et relation salariale) cohérents et acceptables par les acteurs concernés. » (Boyer et Freyssenet, 2000, p.23)
} 
possibles et exploitables de sources de profit » (Ibid, p.18) par différentes modalités d'obtention d'un avantage concurrentiel. Les modèles productifs qui les mettent en œuvre spécifient, d'une part, la nature de la coordination avec la demande par une définition de la qualité des biens que l'entreprise offre sur un marché (politique-produit) et, d'autre part, la forme de coordination qui prévaut à l'intérieur de l'entreprise (organisation productive et relation salariale). Enfin, le compromis de gouvernement porte précisément sur ces trois composantes et ajoutent l'idée que ces moyens mis en œuvre pour l'accumulation doivent être non seulement cohérents et adaptés au mode de croissance (comme l'indique déjà la stratégie de profit), mais aussi « acceptables par les acteurs concernés ».

Du côté de l'EC, dans la continuité de l'étude des formes de coordination générales (Boltanski et Thévenot, 1991), Eymard-Duvernay développe une typologie des entreprises à partir des formes de coordination marchande, industrielle et domestique. L'analyse de ces formes a d'abord été centrée sur l'évaluation des biens (Eymard-Duvernay, 1989). Les différentes façons d'évaluer le travail les discriminent aussi fortement (Eymard-Duvernay, 1990), de même que les différentes règles salariales (Favereau, 2006). Ces règles, étant incomplètes, le contrôle de leur exécution est guidé par une représentation du fonctionnement satisfaisant du collectif formé par les membres de l'entreprise. C'est ici qu'intervient la convention de qualité comme forme spécifiée de convention ${ }_{1}$. L'ensemble des dispositifs dans laquelle cette dernière est ancrée est résumé par la notion de modèle d'entreprise (Eymard-Duvernay, 1987 et 1990). La même logique est à l'œuvre dans une autre typologie conventionnaliste, celle des mondes de production, développée à partir de "l'observation des enchaînements d'actions productives et d'échange » (Salais et Storper, 1993, p.18) plutôt qu'à partir des conflits centrés sur l'évaluation des personnes, qui guident la reconstitution des ordres de grandeurs (Boltanski et Thévenot, 1991).

Il existe une forte correspondance entre les modalités de construction des mondes de production et celles des modèles productifs. Dans les deux cas, le facteur clé de différenciation est le traitement de l'incertitude inhérente au double problème de coordination que doit résoudre l'entreprise, d'une part avec la demande et d'autre part dans l'activité de production. Dans les deux cas aussi, les institutions privilégient l'accès à certains mondes de production ou modèles productifs (restent sur ce point des différences quant aux modalités de construction et finalités des institutions). Dans les deux cas enfin, mais nous touchons ici à un manque, l'horizon temporel de valorisation du capital est négligé.

\section{Conclusion}

En affirmant, à la suite de Keynes, que la spécificité de l'hétérodoxie est de partir d'un univers radicalement incertain, pluriel ou encore contradictoire, nous avons proposé une définition positive des approches critiques à l'égard du courant dominant en économie. Interroger la manière dont les agents transforment cette incertitude incite à mettre en parallèle les principaux outils théoriques forgés par l'EC et la TR pour rendre compte de la coordination. Ce parallèle souligne d'importantes 
convergences entre ces deux approches : les notions constitutives de rapports sociaux pour la TR et de conventions pour l'EC peuvent toutes deux être conçues de manière générale comme des régularités de comportements et d'attentes. Cette conception est solidaire d'une définition commune des règles institutionnelles comme ancrages objectifs des régularités. Ces similitudes aboutissent à une convergence des deux approches sur les principes de cohérence locale et globale entre les régularités et leurs ancrages - cohérence dont témoignent les notions de modèle productif et de mode de régulation pour la $\mathrm{TR}$, de modèle d'entreprise (ou monde de production) et d'équilibre de règles pour l'EC.

Parce que ces convergences portent sur les postulats de base de chaque approche (incertitude, défaut de coordination et rationalité limitée), leurs notions constitutives (conventions et rapports sociaux) et leur compréhension des institutions, nous pouvons affirmer qu'elles se situent en amont des développements sur lesquels l'EC et la TR s'opposent. Ce socle commun invite à un réexamen approfondi des divergences entre les deux approches et peut servir de première base de comparaison dans un débat élargi aux autres formes d'institutionnalisme hétérodoxe.

\section{Références bibliographiques}

Aglietta M. (1976), Régulation et crises du capitalisme, Calmann-Lévy, Paris.

Aglietta M. et Orléan A. (2002), La monnaie entre violence et confiance, Odile Jacob, Paris.

Allaire, G. et Boyer, R. (eds) (1995), La grande transformation de l'agriculture, lectures conventionnalistes et régulationnistes, INRA, Paris, Economica.

Althusser L. (1965), Pour Marx, La découverte, Paris [1986].

Amable, B. et Palombarini, P. (2005), L'économie n'est pas une sciences morale, Paris, Raisons d'agir, collection Cours et Travaux.

Batifoulier, P. (ed) (2001), Théorie des conventions, Paris, Economica.

Benetti C. et Cartelier J (1980), Marchands, salariat et capitalistes, Maspero, Paris.

Bessy C. et Favereau O. (2003), «Institutions et économie des conventions », Cahier d'économie politique, $\mathrm{n}^{\circ} 44$, p.119-164.

Billaudot B. (1996), L'ordre économique de la société moderne, L’Harmattan, Paris.

Boltanski L. et Thévenot L. (1989), Justesse et Justice dans le travail, Cahiers du CEE n³3, PUF, Paris.

Boltanski L. et Thévenot L. (1991), De la Justification. Les économies de la grandeur, Gallimard, Paris.

Boltanski L. et Chiapello E. (1999), Le nouvel esprit du capitalisme, Gallimard, Paris.

Boyer R. (1986), Théorie de la régulation. Une analyse critique, La découverte, Paris.

Boyer R. (1995), «Secteurs, régions et mode de régulation», in Allaire G. et Boyer R., La grande transformation de l'agriculture: Lectures conventionnalistes et régulationnistes, Economica, Paris.

Boyer, R. (2003), «L'anthropologie économique de Pierre Bourdieu», Actes de la recherche en sciences sociales, $\mathrm{n}^{\circ} 150$, décembre, pp.65-78.

Boyer R. (2004), Théorie de la régulation, 1. Les fondamentaux, La découverte, Paris. 
Boyer R. (2004b), Une théorie du capitalisme est-elle possible ? Odile Jacob, Paris.

Boyer R. et Freyssenet M. (2000), Les modèles productifs, La découverte, Paris.

Boyer, R., et Orléan, A. (1994), «Persistance et changement des conventions. Deux modèles simples et quelques illustrations ", in Orléan, A. (ed), Analyse économique des conventions, Paris, PUF, pp.219-247.

Boyer R. et Saillard Y. (2002), Théorie de la régulation. L'état des savoirs, La découverte, Paris.

Coriat, B. (1994), « La théorie de la régulation. Origines, spécificités et perspectives », in Sebaî, F. et Vercellone, C. (eds), Ecole de la régulation et critique de la raison économique, Futur Antérieur, Paris, L’Harmattan, pp.71-99.

Dupuy J.-P., Eymard-Duvernay F., Favereau O., Orléan A., Salais R. et Thévenot L. (1989) «Introduction » au numéro spécial Économie des conventions, Revue économique, vol. 40, n², mars, p. $141-145$.

Eymard-Duvernay F. (1987), Entreprises et produits, Cahiers du CEE n³0, PUF, Paris.

Eymard-Duvernay F. (1989), «Conventions de qualité et formes de coordination», Revue économique, vol 40, n², p.329-360.

Eymard-Duvernay F. (1990), « Modèles d'entreprises et ajustement des politiques d'emploi », La lettre d'information du CEE, $\mathrm{n}^{\circ} 16$.

Eymard-Duvernay F. (1999), «Les compétences des acteurs dans les réseaux», in Callon, M., Cohendet P., Curien N., Dalle J.-M., Eymard-Duvernay F., Foray D. et Schenk E., Réseaux et coordination, Economica, Paris, p.153-189.

Eymard-Duvernay F. (2006), «Introduction », in Eymard-Duvernay F., L'économie des conventions, méthodes et résultats, tome 2 Développements, La découverte, Paris, p.11-16.

Eymard-Duvernay F., Favereau O., Orléan A., Salais R. et Thévenot L. (2006), « Valeur, coordination et rationalité : trois thèmes mis en relation par l'économie des conventions ", in Eymard-Duvernay F., L'économie des conventions, méthodes et résultats, tome 1 Débats, La découverte, Paris, p.23-44.

Favereau O. (1985), «L'incertain dans la «révolution keynésienne » : l'hypothèse Wittgenstein », in Économies et sociétés : série PE (Economia), n³, p. 29-72.

Favereau O. (1986), «La formalisation du rôle des conventions dans l'allocation des ressources », in Salais R. et Thévenot L., Le Travail : marchés, règles, conventions, INSEE - Economica, Paris, p. 249-267.

Favereau O. (1988), «La «Théorie Générale»: de l'économie conventionnelle à l'économie des conventions », Cahier d'économie politique, n¹4-15, p.197-220.

Favereau O. (1989), « Marchés internes, marchés externes », Revue économique, vol 40, n², p.273328.

Favereau, O. (1995), "Conventions et Régulation », Chap. 52 de: Boyer R., et Saillard Y. (eds), Théorie de la Régulation : état des savoirs, Paris, La Découverte, pp.511-520.

Favereau O. (2001), «L'économie du sociologue ou : penser (l'orthodoxie) à partir de Pierre Bourdieu », in Lahire, B., Le travail sociologique de Pierre Bourdieu. Dettes et critiques., édition revue et augmentée, La découverte, Paris.

Favereau O. (2005), "Quand les parallèles se rencontrent: Keynes et Wittgenstein, l'économie et la philosophie », Revue de métaphysique et de morale, $\mathrm{n}^{\circ} 3$, juillet 2005, p.403-427.

Favereau O. (2006), "L'économie des conventions et la théorie des salaires », paru en italien dans Sociologia del Lavaro, numéro spécial consacré à l'économie des conventions, nº102. 
Favereau O. et Thévenot L. (1994), «Réflexions sur une notion d'équilibre utilisable dans une économie de marchés et d'organisations. ", in Ballot G., Les marchés internes du travail : de la microéconomie à la macroéconomie, PUF, Paris, p.273-313.

Keynes J. M. (1936), Théorie générale de l'emploi, de l'intérêt et de la monnaie, traduction française, 1968, Payot, Paris.

Koumakhov R. (2006), «Herbert Simon et l'économie des conventions », in Eymard-Duvernay F., L'économie des conventions, méthodes et résultats, tome 1 Débats, La découverte, Paris, p.197-210.

Lordon, F. (1997), Les quadratures de la politique économique, Les infortunes de la vertu, Paris, Albin Michel.

Lipietz A. (1979), Crise et inflation : pourquoi ?, Maspéro, Paris.

Lipietz A. (1985), « Réflexions autour d'une fable. Pour un statut marxiste des concepts de régulation et d'accumulation », Couverture orange du CEPREMAP n ${ }^{\circ} 8530$.

Lipietz, A. (1995), « De la régulation aux conventions : Le grand bond en arrière ?», Théorie de la régulation et théorie des conventions, Actuel Marx, $\mathrm{n}^{\circ} 17$, premier semestre, pp.39-48.

Lordon, F. (2003), «Conatus et Institutions : pour un structuralisme énergétique », in L'année de la régulation 2003, 7, Paris, Presse de Science Po, pp. 111-146.

Orléan A. (1988), «L'auto-référence dans la théorie keynésienne de la spéculation », Cahier d'économie politique, $\mathrm{n}^{\circ}$ 14-15, Paris, L'Harmattan, pp.229-242.

Orléan, A. (2004), «L'économie des conventions: définitions et résultats. », in Orléan, A. (ed), Analyse économique des conventions, deuxième édition, Paris, PUF.

Palombarini, S. (2001), La rupture du compromis social italien: un essai de macroéconomie politique, Paris, CNRS éditions.

Postel, N. et Sobel, R. (2006), "Quelle théorie hétérodoxe de l'acteur économique ? », in F. EymardDuvernay et O. Favereau (eds.), Conventions et institutions: approfondissements théoriques et contributions au débat politique, à paraître aux éditions La Découverte.

Reynaud, B. (2004), Les règles économiques et leurs usages, Paris, Odile Jacob.

Salais R. et Storper M. (1993), Les mondes de production, enquête sur l'identité économique de la France, Editions de l'EHESS, Paris.

Salais, R., Chatel, E. et Rivaud-Danset, D. (eds) (1998), Institutions et conventions, La réflexivité de l'action économique, Paris, Editions de l'EHESS, collection Raisons Pratiques.

Servais, O. (2000), «Les modèles d'action dans la théorie de la régulation. Habitus, rationalité, routine », Economies et Sociétés, Tome XXXIV, n ${ }^{\circ}$, Janv. 2000, Série Théorie de la régulation, R, n $11, \mathrm{pp} .145-182$.

Théret B., (1992), Régimes économiques de l'ordre politique, PUF, Paris.

Thévenot L. (1985), «Les investissements de forme », in Conventions économiques, Cahiers du CEE, PUF, Paris, p.21-71. 